\title{
Risk Factors Associated with Chronic Kidney Disease of Unknown Origin (Ckdu) in North Central Province (NCP) of Sri Lanka: An Analysis of Evidence to Date
}

\section{Fahim Aslam}

University of Wolverhampton, England, UK

*Corresponding author: Fahim Aslam, University of Wolverhampton, England, UK

Received date: 10 February 2020; Accepted date: 14 February 2020; Published date: 21 February 2020.

Citation: Fahim Aslam (2020) Risk Factors Associated with Chronic Kidney Disease of Unknown Origin (Ckdu) in North Central Province (NCP) of Sri Lanka: An Analysis of Evidence to Date. J Med Case Rep Case Series 1 (1).

Copyright: () 2020 Fahim Aslam. This is an open-access article distributed under the terms of the Creative Commons Attribution License, which permits unrestricted use, distribution, and reproduction in any medium, provided the original author and source are credited

\begin{abstract}
Chronic kidney disease of unknown origin ( $\mathrm{CKDu}$ ) is a global burden among the agricultural communities, this is a non-communicable disease (NCD) which is asymptomatic and irreversible until latter stages of the disease. The disease has no common features unlike chronic kidney diseases (CKD's) making early detection impossible in the patients. The most recent form of CKDu was reported in India, known as the Indian CKDu in late 2010's.

In Sri Lanka, CKDu is highly prevalent in the north central province of the country with nearly $15.1 \%-22.9 \%$ presented with the disease. This region is a dry-zone in which agricultural and farming activities are carried out as the main occupation. Several studies have been carried out linking CKDu to various factors such as heavy metals in water, agrochemicals, heat, dehydration and socio-demographics in NCP. Despite several researches being conducted none of them were able to prove the root cause and causative factors of the disease. Using the available articles online, studies from countries such as India, Nicaragua, Sri Lanka and South America were chosen in which heat stress, dehydration, heavy metal involvement, agrochemicals were common causative factors reported in these geographical locations.

Several studies analyzed indicate that the affected CKDu population were part of the agricultural community in rural areas with less or no proper high school education and family history with CKDu. Recent findings do suggest that a combination study involving socio-demographical data and geographical data will help to end the CKDu debate worldwide and provide new insights into early diagnosis.
\end{abstract}

Keywords: $\mathrm{CKDu}$; $\mathrm{CKD}$; NCP; NCD

\section{Introduction}

Chronic kidney disease's is one of the growing health concerns globally, it is ranked as the $12^{\text {th }}$ most common cause of death by the Global Burden of Disease Study and a reported 1.1 million patients die yearly due to kidney failure and a reported 5-10 million people die due to any form of CKD. Due to the disease having a major impact on economic burden towards the country the treatment and management of the disease has been limited in several low- and middle-income countries such as India, Egypt, Nicaragua, El Salvador and Sri Lanka [1].

CKD's have general features of diabetes mellitus, hypertension and high blood pressure presented in most cases; a new form of the disease also known as chronic kidney disease of unknown origin $(\mathrm{CKDu})$ contains no general features as in CKD. The disease is most common among the low-middle income countries and among the farming population of rural regions. As there is no universal features presented by the disease, early diagnosis cannot be made and the patient prognosis is generally poor as its irreversible.

The first case of CKDu was reported back in 1912, also known as the Itai-itai disease in Japan which commonly affected postmenopausal women, the etiology was unknown until 1968 where cadmium was identified as the main causative agent. Balkan endemic nephropathy (BEN) was reported in the 1950's which was the next type of CKDu identified until the causative agent was identified as Aristolochia species in 1993. The more recent forms of CKDu, Mesoamerican nephropathy (MEN), SL CKDu and Indian CKDu the causative factors are still unidentified and show common similarities between them [2].

Disease being asymptomatic until end-stage-renal-disease (ESRD), severity higher in males than females, main occupation as farming are the common resemblances between MEN, SL-CKDu and Indian-CKDu. Several studies have been conducted over the past decade to identify the root cause for the Sri Lankan
$\mathrm{CKDu}$, environmental and occupational hazards have been identified as the most common features presented among MEN, Indian-CKDu and Sri Lankan CKDu. Heavy metal contamination, use of agrochemicals, heat and dehydration, snake bites and occupational health hazards have been identified as the potential causes of CKDu. However, the underlying etiologies have not been identified in most places such as the NCP of Sri Lanka. By analyzing the available evidence from existing literature papers, the potential causative factors can be identified and provide better insights into Sri Lankan CKDu [3].

\section{Methodology}

Read by QxMD tool was used to search for the keywords "CKDu", "CKD", "North Central Province", "heavy metals", "MEN", "e-GFR", "agrochemicals" were combined with "Sri Lanka", "Lanka" and "SL". Articles were checked on Google scholar for citation scores and most recent papers released, based on that 36 articles were chosen for the study consisting of review papers, editorial, preliminary studies conducted recently and research papers. Relevant information from the papers were summarized and extracted to identify the main causative agents of CKDu in the region to fulfill the study objective.

\section{Background}

Geographical Setting North central province of Sri Lanka is also known as the dry zone of the country with temperature levels ranging from $24.7^{\circ} \mathrm{C}$ to $28.7^{\circ} \mathrm{C}$ in the region and rainfall levels of 1368 mmover the year. The region consists of two main districts, Anuradhapura and Polonnaruwa with an estimated 1.3 million people living in the region with the majority being Buddhists. An estimated2888 villages are in the NCP and $30 \%$ of the income is generated through agricultural 
activities carried out in the region which significantly contributes to the overall GDP of the country $[4,5]$.

\section{Socio-demographical Factors}

The region has agriculture as the main occupation, hence more male workers are heavily involved than the females. The working age group in the region lies around second decade and upwards in general with a minimum of 6-8 hours of paddy harvesting and ploughing taking place during the seasonal period. Educational levels of the individuals of the NCP are lower than most parts of the country where only a reported $25 \%$ of individuals have passed $\mathrm{O} / \mathrm{L}$ and A/L together [6]. Majority of the families contain more than 2 household members out of which at least 1 is affected by CKDu. The average income by the individuals present in the region lies between 3000-10000LKR and are heavily reliant on the agriculture and harvest of paddy crops [6,7].

Impact of agrochemicals on CKDu Agrochemicals are widely used in most South Asian countries to provide a better yield in crop production, Sri Lanka also uses different forms of these such as pesticides, herbicides, insecticides and fertilizers commonly. The dry zone of Sri Lanka uses agrochemicals in high yields, where a study conducted by [8], the efficiency of cost-usage ratio was 0.37 in this region indicating an overuse of agrochemicals. In Sri Lanka, fertilizers are subsidized at high levels making it available for a cheaper rate for many farmers to use higher than recommended amounts. Due to this excessive usage of agrochemicals, the water cycle and the food chain patterns in the region also undergo changes $[\mathbf{9 , 1 0}]$. Most farmers lack education and training to create awareness about the use of agrochemicals, this results in majority of them being directly exposed to the chemicals without any protection. Studies conducted previously has shown agrochemicals can enter the body through nails and hairs leading to skin diseases and cause damage to various organs in the body due to high metal content [11]. Excessive agrochemicals get washed away from the soil leading to excessive nutrients available for micro-organisms such as algae, bacteria and fungi to grow and incorporate themselves on the crops which can directly affect the food cycle chain of humans. Lack of water available in the region leads to farmers relying on well and spring water sources for consumption, the agrochemicals washed away from the soil can leech into the waterway pathways leading to groundwater consumption being contaminated. Phosphorus is identified as one of the key elements that causes "eutrophication" of the water quality and increases the risk of water drying out in the region [12]. Nitrogen is another element that harms the ecosystem, excessive nitrogen can lead to over stimulation of aquatic plants and algae formation which competes for oxygen with other organisms. Several studies have shown that many fish species are contaminated with high heavy metal content and unable to be consumed by the residents of the NCP, with increasing damage to the ecosystem the quantity of fish present in the region will begin to reach its extinction with time [13]. Excessive nitrogen can also cause increase in pollutants present in the region; ammonia is one of the greenhouse gases formed due to excessive nitrogen present and this decreases the air quality leading to problems such as asthma and respiratory tract infections [14].

Potassium is the most important element needed for plant growth, where it allows movement of nutrients and water into the plants and needed for ATP production to regulate photosynthesis. Lack of potassium can lead to plant defects being visible and also can affect the growth of the plant, most farmers use "potash" a type of potassium fertilizer that is expensive and needed as an essential component during harvesting. As the subsidies are lowered in South Asian countries, the potassium fertilizers are used excessively causing draining of potassium into groundwater. This could lead to hyperkalemia, which is a common condition presented among CKD individuals and lead to kidney dysfunction with time [15].
A combination of three essential elements can lead to long-term harm to the ecosystem, environment and human health, the impact of agrochemicals could be a probable cause of CKDu which has not been proven yet.

Heavy metals involvement in CKDu Several heavy metals have been linked to be causative agents for $\mathrm{CKDu}$, these heavy metals are present as constituents and waste products of the agrochemicals. Arsenic is one of main heavymetals present in triple super phosphate (TSP) which is a common fertilizer used by farmers for the cultivation of rice. As stated by Rawat [16] nearly $2100 \mathrm{~kg}$ of arsenic is imported yearly in the form of TSP fertilizers.

Despite the fact that the WHO considered pesticides containing arsenic as hazardous many farmers still continue to use them as they are unaware of the harm caused by these heavy metals [17].

Chronic arsenic toxicity (CAT) is a common feature presented among CKDu affected patients, where long term exposure can lead to cancer and impacts cognitive development in young adults. Arsenic contaminated ground water and food can lead to ingestion of these carcinogenic particles that can lead to long term effects as kidneys acts as the organs that helps to remove the waste products from the body. This can lead to long term irreversible damage caused to the kidneys leading to fibrosis and increased proteinuria levels in the patients' blood leading to nephrotoxin build up inside the body [18].

Cadmium is another heavy metal that is present in phosphorous fertilizers which is a carcinogenic compound for human health. Several countries have banned fertilizers containing cadmium due to the direct impact it has on the kidneys. European Union passed a bill to limit the use of cadmium containing fertilizers as its adverse effects were causing increased reported incidents of kidney and respiratory system failures due to cadmium exposure [19]. Renal tubular dysfunction and increased creatinine levels in blood and urine are the main features observed in individuals with increased cadmium exposure. This is proven in the NCP of Sri Lanka, where excessive amount of cadmium was presented in ingested food and water which could probably be one of the causes of CKDu in these individuals $[\mathbf{2 0 , 2 1}]$. Rice being the staple food consumed by the residents of the NCP and the cadmium levels found in rice in this region is well above the international limits set by the WHO indicating the excessive usage of agrochemicals in the region. Facts and theories states cadmium has a major impact like arsenic on the kidneys, but till date there has been no evidence that link cadmium to be the direct causative agent of SL-CKDu unlike in "Itai-itai disease" [22].

Glyphosate is another widely used herbicide which has been identified as one of the probable causative factors of $\mathrm{CKDu}$. Exposure to glyphosate in minimal amounts has no or less impact on the body organs or immune system, whereas excessive exposure could lead to severe complications such as DNA damage in exposed individuals. Glyphosate unlike arsenic and cadmium shows minimum levels of the heavy metal presence in the blood or urine and makes slow cellular damage inside the body. Regardless of the fact the WHO deemed glyphosate as "probably carcinogenic", the damage caused by the heavy metal can significantly impact the growth and metabolism in individuals when exposed for a long period of time [23]. In Sri Lanka increased levels of glyphosate is presented in farming lands topsoil indicating prolonged exposure which can be one of the causative agents for CKDu [24].

Arsenic and cadmium are released into the environment by the phosphorous fertilizers and glyphosate is released through herbicides added for the harvesting process. Three of these heavy metals are released from the use of agrochemicals and play a significant part in proliferating damage to the kidneys. The chief contributing causative agent might be unknown, nonetheless the effect of heavy metals on kidneys can be understood. Water contamination as a source of CKDu outbreak Due to the cumulative accumulation of agrochemicals in the region the overall groundwater quality has impacted the overall quality of water. Ions such as 
sodium, fluoride, magnesium, calcium, potassium, lead, ammonium and phosphate are generally present in drinking water in the region [25]. A study conducted using mice with the contaminated drinking water sources indicated several deaths occurring with over consumption of water and formation of necrotic lesions of kidneys and liver [26]. Patients in the NCP are more liable to be affected with gastrointestinal and diarrheal diseases due to this consumption of contaminated water and could possibly cause long term damage to the kidneys as well. Lack of water available in region causes individuals to be reliant on water from groundwater sources such as wells and tanks, even though there are significantly high amounts of heavy metals mixed in the water content people consume them without filtering the heavy metals available [27]. Changes in $\mathrm{pH}$ value and electrochemical gradient net charge of water also impacts this, where at different temperatures in the region the release rate of heavy metals vary, at high temperatures asin the NCP. Increased heavy metals deposition in the body leads to oxygen deprivation to the affected organs, specially the kidneys as the particles get filtered over here. High heavy metal

deposition also leads to problems in homeostasis and osmoregulation in the body which could cause increased dehydration in the body. Several studies [28,29] have stated that the lack of water availability in the region provides the residents with no option but consume ground watersources which contains high levels of contaminants and probable causative factors which can cause progression of CKDu.

Impact of heat and dehydration on CKDu Majority of the workers affected with CKDu have been found to be working at high temperatures for several decades. In South American countries sugarcane plantations and South Asian countries paddy cultivation are the only difference in terms of farming, whereas the working hours and climatic demographics are similar. Due to high intensity work being carried out, a high amount of sodium is lost by the body through sweat, this leads to an imbalance of ions inside the body leading to dehydration and dysuria in the farmers. Dehydration can lead to either farmers consuming excessive contaminated water from closest water source or else remain dehydrated to prevent drinking contaminated water. Either way the kidneys get affected due to the heat stress exerted onto the body leading to symptoms such as headache and dizziness in most instances. Women are liable to be affected due to this as the water retention in their bladder is relatively lower than in males and more liable to be affected by urinary tract infections as well.

A study carried out in 2015 identified heat stress as the main causative factor of CKDu in Mesoamerican nephropathy patients in South America [30] and studies are ongoing currently to prove the hypothesis made by the research team. Farmers have high endurance levels which the body is able to resist any form of heat and pressure exerted by the surroundings leading to acute kidney injury that can develop into chronic injury overtime [31]. Recurrent and prolonged dehydration has been identified as a causative factor that could lead to glomerular hypertension tubular injury due to the oxidative stress exerted onto the kidneys [32] which could be a probable pathway leading to CKDu.

In Sri Lanka, preliminary studies have been conducted to identify the role of heat in CKDu affected individuals, thus far there has been no significant evidence to back the claim of "heat stress induced CKDu".

\section{Discussion}

The most recent findings about CKDu has helped several countries burdened by the disease to bridge the gap between understanding the etiology of the disease and finding the root causative factors. Endemic regions have been identified over the last decade which show similar sociodemographic and climatic factors presented among them, indicating a common causative factor presented among this community. Sri Lanka was chosen as the ideal region to identify and point out the causative factors due to the prevalence of the disease from the 1990's. Several studies have been conducted since then highlighting different causative factors and their probable roles in causing the disease to progress further. North Central Province (NCP) of Sri Lanka is a unique location which supports all"suggested hypotheses" worldwide and shows incident rates in the region. Change in agricultural practices and providing an alternative way of farming will increase the likelihood of farmers being less exposed by the disease, although these methods have been suggested there has been no continuous monitoring carried out which questions the reliability of the methods $[\mathbf{3 3}, \mathbf{3 4}]$. A study carried out by the World Health Organization in 2014 stated that residents of the NCP exposed to agricultural activities are at high risk of contracting CKDu if protective measures are not taken [35]. In Sri Lanka a reported 30,000 individuals are reported tobe affected by $\mathrm{CKDu}$ and many more unreported or undiagnosed cases are still present in the country [7].

Most recently the disease has been identified in Andhra Pradesh of India in 2010, where reported patients exhibited same symptoms as in SL-CKDu. Perhaps a global epidemic could rise due to the impact of CKDu and measures need to be taken to address the disease and identify the cause, otherwise the future generations will be burdened by the disease and the overall economy of the country could be affected as well [36]. An increased economic health burden in the country would cause limitations and lack of resources available in treating the patients, especially in India and Sri Lanka due to the free healthcare services provided.

\section{Conclusion}

CKDu is a form of CKD which is a growing concern in the North Central Province (NCP) of Sri Lanka, several hypotheses have been suggested and studies have been conducted widely to understand the impact of the disease. The mortality rate caused by the disease in the region is relatively high and could be having similar impact across other low-income countries as well due to the limitations in outpatient management. Studies needs to be conducted globally across all affected regions using available resources to identify the cause of the disease and must be considered as an urgent problem to be addressed.

\section{Statement}

The mini review focuses on understanding the main causative factors of CKDu in the region and how they can affect the overall health of an individual. Acknowledgement I would like to thank my supervisor, Dr. Nishantha Kumarasinghe and the CKDu-Care unit for providing insights and information about CKDu. I would like to extend my thanks to all the authors who have been working continuously to find the causative factors of the disease and publish them to allow fellow researchers to understand the disease better.

\section{Disclosure Statement}

There are no conflicts of interest in publishing this article, all author(s) involved gave their consent for publication.

\section{Funding}

No funding was obtained for this review

\section{References}

1. Wimalawansa SA, Wimalawansa SJ (2016) Environmentally induced, occupational diseases with emphasis on chronic kidney disease of multifactorial origin affecting tropical countries. Ann Occup Environ Med 28 (1): 33 .

2. Gifford FJ, Gifford RM, Eddleston M, Dhaun N (2017) Endemic Nephropathy Around the World. Kidney Int Rep 2(2): 282-292. 


\section{Journal of Medical Case Reports and Case Series: Open Access}

3. Torres C, Aragón A, González M, López I, Jakobsson K, et al. (2010) Decreased Kidney Function of Unknown Cause in Nicaragua: A CommunityBased Survey. Am J Kidney Dis 55(3): 485-496.

4. Redmon JH, Elledge MF, Womack DS, Wickremashinghe R, Wanigasuriya KP, et al. (2014) Additional perspectives on chronic kidney disease of unknown aetiology (CKDu) in Sri Lanka - lessons learned from the WHO CKDu population prevalence study. BMC Nephrol 15: 125.

5. Abeywardana N, Schütt B, Wagalawatta T, Bebermeier W (2019) Indigenous Agricultural Systems in the Dry Zone of Sri Lanka: Management Transformation Assessment and Sustainability. Sustainability 11: 910.

6. Paranage K (2018) Constructing the 'legalities' of encroachments in dam, canal, and stream reservations in the north-central province of Sri Lanka. Development Studies Research 5: 59-68.

7. Noble A, Amerasinghe P, Manthrithilake H, Arasalingam S (2014) Review of literature on chronic kidney disease of unknown etiology (CKDu) in Sri Lanka.

8. Ruwanpathirana T, Senanayake S, Gunawardana N, Munasinghe A, Ginige S, et al. (2019) Prevalence and risk factors for impaired kidney function in the district of Anuradhapura, Sri Lanka: a cross-sectional populationrepresentative survey in those at risk of chronic kidney disease of unknown aetiology. BMC Public Health 19(1): 763.

9. Allocative Efficiency of Land in Major Irrigation Paddy Farming in Sri Lanka. March 20-21, 2017 London Back HSSE-17 \& LEBIS-17 2017; DOI: 10.17758/uruae. uh0317008.

10. Herath H, Gunawardena E, Wickramasinghe W (2015) The impact of "KethataAruna" fertilizer subsidy programme on fertilizer use and paddy production in Sri Lanka. Tropical Agricultural Research 25: 14.

11. Hamid ZA, Ishak I, Lubis SH, Mohammad N, Othman H, Saat NZM, et al. (2017) Evaluation of Trace Elements in the Nails and Hair of Farmers Exposed to Pesticides and Fertilizers. J Agri Sci 9: 79.

12. Aleksandra Drizo (2019) Water Quality Legislation and Policy for Phosphorus Pollution Control Phosphorus Pollution Control - Policies and Strategies 11-40.

13. Wijesinghe H, Idroos FS, Manage PM (2018) Heavy metal contamination status in seven fish species from reservoirs of Polonnaruwa district, Sri Lanka. Sri Lanka J Aqua Sci 23: 95.

14. Ward M, Jones R, Brender J, Kok TD, Weyer P, et al. (2018) Drinking Water Nitrate and Human Health: An Updated Review. Int Environ Res Public Health 15(7): E1557.

15. Chandrasiri GN, Gunawardena U (2018) Health Damage Cost of Rice Processing Industry of the North Central Province. Proceedings of International Forestry and Environment Symposium 22.

16. Rawat J, Sanwal P, Saxena J (2016) Potassium and Its Role in Sustainable Agriculture. Potassium Solubilizing Microorganisms for Sustainable Agriculture 235-253.

17. Jayasumana C, Fonseka S, Fernando A, Jayalath K, Amarasinghe M, et al. (2015) Phosphate fertilizer is a main source of arsenic in areas affected with chronic kidney disease of unknown etiology in Sri Lanka. Springer Plus 4: $\underline{90 .}$

18. Bencko V, Foong FYL (2017) The history of arsenical pesticides and health risks related to the use of Agent Blue. Ann Agric Environ Med 24: 312-316.

19. Zheng L, Kuo C-C, Fadrowski J, Agnew J, Weaver VM, et al. (2014) Arsenic and Chronic Kidney Disease: A Systematic Review. Curr Environ Health Rep 1: 192-207.

20. Ulrich AE (2019) Cadmium governance in Europes phosphate fertilizers: Not so fast? Science of The Total Environment 650: 541-545,
21. Jayalal TBA (2015) Chronic kidney disease of uncertain aetiology: adding vital piece of information to the national project team report of Sri Lanka. BMC Nephrol 16: 216.

22. Ananda Jayalal TB, Jayaruwan Bandara TWMA, Mahawithanage STC, Wansapala MAJ, Galappaththi SPL (2019) A quantitative analysis of chronic exposure of selected heavy metals in a model diet in a CKD hotspot in Sri Lanka. BMC Nephrol 20(1): 208.

23. Kumar S, Sharma A (2019) Cadmium toxicity: effects on human reproduction and fertility. Rev Environ Health 34: 327-338.

24. Baran MJ, Cibiński M (2014) Glyphosate-based phospho-organic herbicides - an outline of action, metabolism and the selected effects on humans and other organisms. Arch Physiother Global Res 18: 35-45.

25. Gunarathna S, Gunawardana B, Jayaweera M, Manatunge J, Zoysa K (2018) Glyphosate and AMPA of agricultural soil, surface water, groundwater and sediments in areas prevalent with chronic kidney disease of unknown etiology, Sri Lanka. J Environ Sci Health B 53: 729-737.

26. Wanasinghe WCS, Gunarathna MHJP, H. M. P. I. K. Herath, Jayasinghe GY (2018) Drinking Water Quality on Chronic Kidney Disease of Unknown Aetiology (CKDu) in Ulagalla Cascade, Sri Lanka. Sabaragamuwa University Journal 16: 17.

27. Wasana HMS, Perera GDRK, Gunawardena PDS, Fernando PS, Bandara J: WHO water quality standards Vs Synergic effect(s) of fluoride, heavy metals and hardness in drinking water on kidney tissues. Sci Rep 7:42516.

28. Li H, Shi A, Li M, Zhang X (2013) Effect of pH, Temperature, Dissolved Oxygen, and Flow Rate of Overlying Water on Heavy Metals Release from Storm Sewer Sediments. J Chem 2013: 1-11.

29. Chandrakumara D (2013) Human capital formation within families: a study in the North Central Province of Sri Lanka. Sri Lanka J Soc Sci 33: 47.

30. Sunil J Wimalawansa (2015) Agrochemicals and Chronic Kidney Disease of Multi-Factorial Origin: Environmentally Induced Occupational Exposure an Occupational Exposure Disease. Int J Nephrol Kidney Fail 1.

31. García-Trabanino R, Jarquín E, Wesseling C, Johnson RJ, González-Quiroz $\underline{M}$, et al. (2015) Heat stress, dehydration, and kidney function in sugarcane cutters in El Salvador - A cross-shift study of workers at risk of Mesoamerican nephropathy. Environmental Research 142: 746-755.

32. Rojas-Valverde D, Olcina G, Gutiérrez-Vargas R, Crowe J (2019) Heat Strain, External Workload, and Chronic Kidney Disease in Tropical Settings: Are Endurance Athletes Exposed? Front Physiol 10:1403.

33. Roncal-Jimenez C, Lanaspa M, Jensen T, Sanchez-Lozada L, Johnson R (2015) Mechanisms by Which Dehydration May Lead to Chronic Kidney Disease. Ann Nutr Metab 66: 10-13.

34. Jayasekara K, Kulasooriya P, Wijayasiri K, Rajapakse E, Dulshika D, et al. (2019) Relevance of heat stress and dehydration to chronic kidney disease (CKDu) in Sri Lanka. Prev Med Rep 15: 100928.

35. Jayatilake N, Mendis S, Maheepala P, Mehta FR (2013) Chronic kidney disease of uncertain aetiology: prevalence and causative factors in a developing country. BMC Nephrol 14: 180.

36. Redmon JH, Elledge MF, Womack DS, Wickremashinghe R, Wanigasuriya $\underline{K P}$, et al. (2014) Additional perspectives on chronic kidney disease of unknown aetiology $(\mathrm{CKDu})$ in Sri Lanka - lessons learned from the WHO CKDu population prevalence study. BMC Nephrol 15: 125. 